\title{
Toward a guided approach to platelet activation in diabetes
}

\author{
M. D. Linden
}

Published online: 5 December 2012

(C) Springer Science+Business Media New York 2012

Diabetes is associated with substantially poorer cardiovascular outcomes. Furthermore, people with diabetes are at greatly increased risk of not responding to standard antiplatelet therapy for acute coronary syndromes, leading to higher mortality and stent thrombosis [1]. Poor glucose control likely contributes to these cardiovascular events through changes in platelet membrane fluidity and signalling, increased production of platelets leading to a greater fraction of younger and more reactive platelets not yet exposed to antiplatelet therapy, and increased triggering of platelet activation arising from an underlying thromboinflammatory milieu [2].

While patients with higher thrombotic risk may be prospectively identified through laboratory identification of elevated blood glucose or HbA1c [3], the approach to treatment for diabetic platelet hyper-responsiveness remains unclear. Options such as increasing frequency of antiplatelet therapy, additional and more aggressive antiplatelet therapy or more intensive glucose control have been suggested (Fig. 1). However, the data to support these are limited and, particularly where more aggressive antiplatelet therapy is proposed, must be measured against the potential for increased bleeding risk in patients with hyperglycemia.

In patients with type-2 diabetes, Capodanno et al. [4] demonstrated enhanced inhibition of platelet function when patients took $81 \mathrm{mg}$ of aspirin twice per day compared with $81 \mathrm{mg}$ daily. Spectre et al. [5] showed greater inhibition of platelet function with $75 \mathrm{mg}$ twice per day when compared

M. D. Linden $(\square)$

Centre for Microscopy, Characterisation and Analysis, M510, University of Western Australia, 35 Stirling Highway, Nedlands, WA 6009, Australia

e-mail: matthew.linden@uwa.edu.au to $75 \mathrm{mg}$ daily in patients with type-2 diabetes and vascular complications. In a recent study Rocca et al. [6] showed that increasing frequency of dose better attenuated platelet activation over $24 \mathrm{~h}$ in both diabetic and non-diabetic patients. Therefore increasing the frequency of antiplatelet therapy dosing represents an appealing strategy in patients with diabetes and poor glycemic control. However, the clinical effectiveness of this approach on cardiovascular outcome, as well as the net benefit against increased bleeding risk remains to be investigated. Similarly, the addition of more aggressive antiplatelet therapy, such as newer ADP receptor antagonists with greater potency and bioavailability, or emerging thrombin receptor antagonists have been suggested [2]. The use of these must be weighed against the potentially increased risk of bleeding.

While altering antiplatelet therapy strategies in patients with diabetes and poor glycemic control is an appealing avenue for further clinical investigation, a potential alternative or complimentary approach might be to achieve improved glycemic control in order to attenuate platelet activation. Several studies have assessed the ability of infused insulin in patients with diabetes to attenuate platelet function and improve cardiovascular outcome in acute coronary syndromes [7]. While evidence of improvement in cardiovascular outcome is variable, intensive treatment with insulin in patients with diabetes may attenuate platelet activation relative to conventional approaches to glycemic control [8].

In this issue of the Journal of Thrombosis and Thrombolysis, Vivas et al. [9] explore whether tight glycemic control with insulin infusion attenuates platelet reactivity in patients with an acute coronary syndrome and hyperglycemia at admission. In a post hoc analysis of the prospective, randomized, open-label CHIPs study, 67 patients with poor glycemic control (as measured by HbA1c $>6.5 \%$ ) were 
Fig. 1 Contributing mechanisms and potential approach to treatment of diabetes associated platelet activation

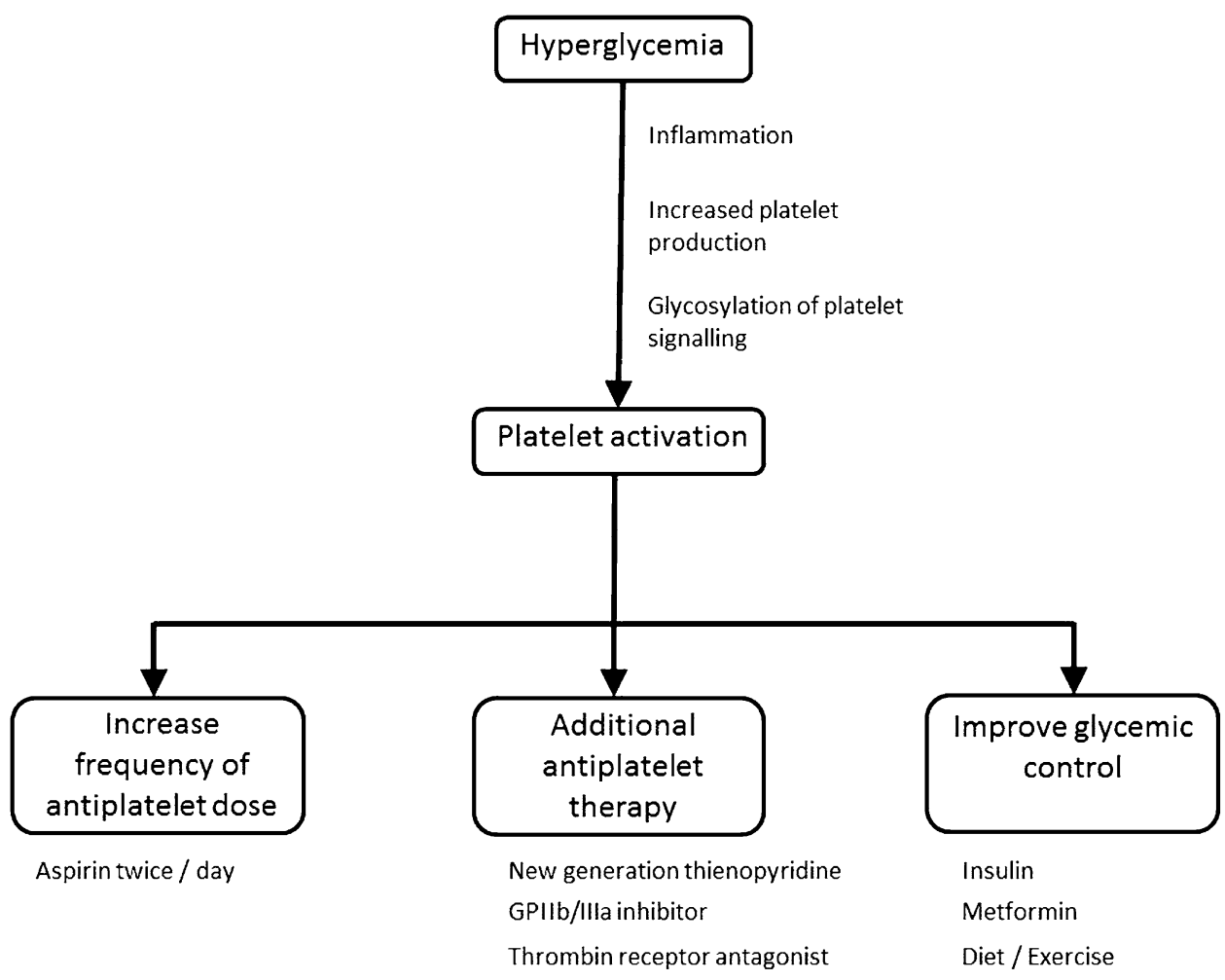

While significant platelet activation might be expected in this cohort, the magnitude of platelet activation (e.g. $\sim 45 \%$ P-selectin expression at admission) reported by Vivas et al. is higher than previously reported for acute coronary syndromes [10]. This may reflect pre-analytical variables resulting from the challenges of platelet function testing in the clinical setting. However, the magnitude of difference in parameters of platelet reactivity between conventional and intensive treatment groups was quite substantial (e.g. $14.3 \%$ higher $20 \mu \mathrm{M}$ ADP stimulated platelet aggregation in the conventional group) and likely to be of clinical significance.

The mechanism by which better control of hyperglycemia contributes to attenuated platelet function in this study are unclear and likely to be multifactorial. It is hypothesised that stimulation of the IRS-1 receptor may be involved, and this is suggested by Vivas et al. However, these data show augmentation of responsiveness to chemical stimulation with conventional therapy, rather than elevated circulating platelet activation by IRS-1. However, this may reflect a lack of statistical power to demonstrate a difference in unstimulated PAC-1 or CD62P, rather than an absence of effect.

Of interest, in a separate analysis of 42 patients also with acute coronary syndrome and hyperglycemia at admission, but better glucose control (as measured by HbAlc $<6.5 \%$ ), Vivas et al. report no improvement in parameters of platelet function at discharge with intensive glucose control strategy 
( $n=20)$ when compared to the conventional treatment $(n=22)$. The effect size between HbA1c $>6.5 \%$ and HbA1c $<6.5 \%$ groups was not directly compared and consequently the data is insufficient to support a conclusion of a difference between platelet response to insulin therapy between those with and without elevated HbA1c. Nevertheless, this paper highlights the potential benefit of tailored therapy in response to laboratory monitoring. This paper further underscores the need for clinical research evaluating a guided approach to the management of platelet function through tighter glucose control in subsets of patients on the basis of laboratory assessment of elevated HbAlc. While preliminary, the research presented by Vivas, et al. therefore represents an important seminal finding which will likely inform further prospective studies of this guided approach to therapy. Future investigation should focus on whether the improvements in platelet reactivity achieved by adjusting approaches to antiplatelet or glucose limiting therapies translates to improved clinical outcomes.

\section{References}

1. Pignone M, Alberts MJ, Colwell JA, Cushman M, Inzucchi SE, Mukherjee D, Rosenson RS, Williams CD, Wilson PW, Kirkman MS (2010) Aspirin for primary prevention of cardiovascular events in people with diabetes: a position statement of the American Diabetes Association, a scientific statement of the American Heart Association, and an expert consensus document of the American College of Cardiology Foundation. Circulation 121(24):2694-2701

2. Linden MD, Tran HA (2012) Overcoming aspirin treatment failure in diabetes. Crit Rev Clin Lab Sci. doi:10.3109/10408363. 2012.731377

3. Hadjadj S, Coisne D, Mauco G, Ragot S, Duengler F, Sosner P, Torremocha F, Herpin D, Marechaud R (2004) Prognostic value of admission plasma glucose and $\mathrm{HbA}$ in acute myocardial infarction. Diabet Med 21(4):305-310

4. Capodanno D, Patel A, Dharmashankar K, Ferreiro JL, Ueno M, Kodali M, Tomasello SD, Capranzano P, Seecheran N, Darlington A, Tello-Montoliu A, Desai B, Bass TA, Angiolillo DJ (2011) Pharmacodynamic effects of different aspirin dosing regimens in type 2 diabetes mellitus patients with coronary artery disease. Circ Cardiovasc Interv 4(2):180-187

5. Spectre G, Arnetz L, Ostenson CG, Brismar K, Li N, Hjemdahl P (2011) Twice daily dosing of aspirin improves platelet inhibition in whole blood in patients with type 2 diabetes mellitus and micro- or macro-vascular complications. Thromb Haemost 106(3):491-499

6. Rocca B, Santilli F, Pitocco D, Mucci L, Petrucci G, Vitacolonna E, Lattanzio S, Mattoscio D, Zaccardi F, Liani R, Vazzana N, Del Ponte A, Ferrante E, Martini F, Cardillo C, Morosetti R, Mirabella M, Ghirlanda G, Davi G, Patrono C (2012) The recovery of platelet cyclooxygenase activity explains interindividual variability in responsiveness to low-dose aspirin in patients with and without diabetes. J Thromb Haemost 10(7):1220-1230

7. Wiener RS, Wiener DC, Larson RJ (2008) Benefits and risks of tight glucose control in critically ill adults: a meta-analysis. JAMA 8:933-944

8. Vivas D, Garcia-Rubira JC, Bernardo E, Angiolillo DJ, Martin P, Calle-Pascual A, Nunez-Gil I, Macaya C, Fernandez-Ortiz A (2011) Effects of intensive glucose control on platelet reactivity in patients with acute coronary syndromes. Results of the CHIPS Study ("Control de Hiperglucemia y Actividad Plaquetaria en Pacientes con Sindrome Coronario Agudo"). Heart 97(10):803-809

9. Vivas D, Garcia-Rubira JC, Bernardo E, Angiolillo DJ, Martin P, Calle-Pascual A, Nunez-Gil I, Macaya C, Fernandez-Ortiz A (2012) Influence of HbA1c levels on platelet function profiles associated with tight glycemic control in patients presenting with hyperglycemia and an acute coronary syndrome : a subanalysis of the CHIPS Study ("Control de HI perglucemia y Actividad P laquetaria en Pacientes con S indrome Coronario Agudo"). J Thromb Thrombolysis. doi:10.1007/s11239-012-0834-3

10. Linden MD, Furman MI, Frelinger AL 3rd, Fox ML, Barnard MR, Li Y, Przyklenk K, Michelson AD (2007) Indices of platelet activation and the stability of coronary artery disease. J Thromb Haemost 5(4):761-765 